\title{
The 1-Minute Sit-to-Stand Test in Adults With Cystic Fibrosis: Correlations With Cardiopulmonary Exercise Test, 6-Minute Walk Test, and Quadriceps Strength
}

\author{
Mathieu Gruet PhD, Leonardo Alexandre Peyré-Tartaruga PhD, Laurent Mely MD, and \\ Jean-Marc Vallier MD PhD
}

\begin{abstract}
BACKGROUND: Exercise testing is part of the regular assessment of patients with cystic fibrosis (CF). We aimed to evaluate (1) the convergent validity of the 1-min sit-to-stand (STS) test in CF by investigating its relationships with peak oxygen uptake (peak $\dot{\mathrm{V}}_{\mathrm{O}_{2}}$ ), quadriceps strength, and quality of life and (2) to compare these associations with those of the 6-min walk test (6MWT). METHODS: Twenty-five adults with $\mathrm{CF}\left(\mathrm{FEV}_{1}=59 \pm 24 \%\right)$ performed the STS test, the 6MWT, quadriceps strength assessment, and cardiopulmonary exercise test (CPET). Physical activity level, quality of life, and self-esteem were assessed by questionnaires. RESULTS: STS repetitions, 6-min walk distance, quadriceps strength, and peak $\dot{\mathrm{V}}_{\mathrm{O}_{2}}$ were, respectively, $71 \pm 12,90 \pm 10,93 \pm 29$, and $62 \pm 16 \%$ of predicted. The STS test had moderate associations with peak $\dot{V}_{\mathrm{O}_{2}}(\mathrm{r}=0.56, P=.004)$, quadriceps strength $(r=0.52, P=.008)$, and some questionnaire items (eg, perceived physical strength, $r=0.67, P<.001)$ only when repetitions were expressed as a product of body weight. Overall, these associations were weaker than those obtained from 6-min walk distance $\times$ weight. Oxygen desaturation during the STS test was strongly associated with oxygen desaturation during CPET $(r=0.80, P<.001)$. Peak heart rate was lower during the STS test as compared with CPET $(P<.001)$ and the 6MWT $(P=.009)$. CONCLUSIONS: The STS test cannot be used as a replacement for CPET to accurately assess peak exercise capacity in CF. The STS test may have utility in detecting patients with CF who may exhibit a high level of oxygen desaturation during heavy exercise. Further studies should identify the factors contributing to STS performance to confirm the potential interest of STS repetitions $\times$ body weight outcome as a useful submaximal exercise parameter in CF. Key words: exercise test; cystic fibrosis; peak oxygen uptake; limb muscle function; quality of life; rehabilitation. [Respir Care 2016;61(12):1620-1628. ( 2016 Daedalus Enterprises]
\end{abstract}

\section{Introduction}

Cystic fibrosis $(\mathrm{CF})$ is one of the most widespread lifeshortening genetic diseases. It is a progressive multisystem

\footnotetext{
Drs Gruet, Mely, and Vallier are affiliated with Université de Nice Sophia Antipolis, Université de Toulon, Laboratoire Motricité Humaine Education Sport Santé, Toulon, France. Dr Peyré-Tartaruga is affiliated with the Exercise Research Laboratory, Universidade Federal do Rio Grande do Sul, Porto Alegre, Brazil. Dr Mely is affiliated with the Centre de Ressources et de Compétences de la Mucoviscidose (CRCM), Hôpital Renée Sabran, Giens, France.

The authors have disclosed no conflicts of interest.

Supplementary material related to this paper is available at http:// www.rcjournal.com.
}

disorder, predominantly affecting the lungs and the digestive system. Despite considerable advances in therapies, there is no curative treatment for CF. Improvements in patients' function, well-being, and longevity are thus important clinical goals. Regular exercise testing is now strongly recommended as part of the care for patients with CF. ${ }^{1,2}$ Exercise intolerance is a hallmark of CF. Exercise testing is needed to detect physical limitation, the associated underlying factors, and potential adverse effects of

Correspondence: Mathieu Gruet PhD, LAMHESS EA 6312, Université de Toulon, BP 20132, 83957 La Garde, France. E-mail: gruet@ univ-tln.fr.

DOI: $10.4187 /$ respcare. 04821 
exercise. ${ }^{1,3}$ It is also used to set the intensity of exercise in training programs on an individualized basis. ${ }^{1}$ Importantly, exercise testing has a strong prognostic value in $\mathrm{CF},{ }^{4}$ and reduced aerobic exercise capacity has been cross-sectionally and longitudinally associated with reduced health-related quality of life (HRQOL). ${ }^{5}$

A cardiopulmonary exercise test (CPET) with gas exchange measurements is the accepted standard exercise test, with peak oxygen uptake (peak $\dot{\mathrm{V}}_{\mathrm{O}_{2}}$ ) as the primary outcome. However, many centers do not have the resources to include CPET on a routine basis, ${ }^{6}$ mainly because CPET equipment is costly, labor intensive, and requires trained staff for supervision and interpretation. Hence, various simple field tests are often used as an alternative for exercise assessment in CF. The most commonly applied field tests to measure exercise capacity in CF are self-paced timed walking tests. The 6-min walk test (6MWT) is among the most popular field test in CF in both clinical and research settings. This test is reliable ${ }^{7}$ and has prognostic value in patients with moderate to severe CF. ${ }^{8}$ However, the $6 \mathrm{MWT}$ requires substantial space (ie, $30-40 \mathrm{~m}$ ) and provides only limited information on limb muscle function, an important determinant of exercise tolerance ${ }^{9,10}$ and HRQOL in CF. ${ }^{11}$

The 1-min sit-to-stand (STS) test is a rapid and simple test that is increasingly used in respiratory diseases such as COPD. ${ }^{12-14}$ The STS movement is frequently performed in daily living and is thought to be a measure of lower-limb strength. The STS test has been recently demonstrated to be reliable and sensitive to intervention in a small sample of adults with CF. ${ }^{15}$ Similarly to the 6MWT, this test elicits submaximal responses in the majority of patients with $\mathrm{CF}$ and thus may not reflect the integrated response of the organism to exercise nor permit identification of the factors limiting exercise tolerance. To be of clinical value, the STS test should demonstrate high convergent validity with important outcomes of peak aerobic fitness (eg, peak $\dot{\mathrm{V}}_{\mathrm{O}_{2}}$, peak work rate) but also with measures of patient well-being (eg, HRQOL, self-esteem). If the STS test is intended to be used when CPET is not available, then its associations with these outcomes should be of comparable strength compared with those obtained from other commonly used field tests (eg, 6MWT). Due to its simplicity, the STS test may thus, for instance, be suitable for repeated measurements during the course of pulmonary rehabilitation. Whether this test may detect important information, such as oxygen desaturation, also requires investigation. Finally, it would be of value to determine whether the STS test can be used as a measure of lowerlimb muscle strength in CF.

The primary aim of this study was to investigate the relationship of the STS test with important physiological and psychological outcomes in adults with CF (ie, peak $\dot{\mathrm{V}}_{\mathrm{O}_{2}}$, quadriceps strength, HRQOL, and self-esteem) and to compare these associations with those of the 6MWT. Pre-

\section{QUICK LOOK}

\section{Current knowledge}

Exercise testing is part of the regular assessment of patients with cystic fibrosis (CF). Cardiopulmonary exercise test is the accepted standard exercise test, but many centers do not have the resources to include this test on a routine basis. Simple field tests are needed as an alternative to assess the functional capacity of patients with $\mathrm{CF}$.

\section{What this paper contributes to our knowledge}

The 1-min sit-to-stand test was performed in adults with $\mathrm{CF}$. This test cannot be used to accurately predict peak exercise tolerance in CF but may provide a submaximal alternative to the usual exercise tests which are not always available in CF centers or cannot be performed on a regular basis. The 1-min sit-to-stand test was able to detect subjects with CF who exhibited a high level of oxygen desaturation during heavy exercise.

vious studies in COPD showed that the association between 6MWT and peak $\dot{\mathrm{V}}_{\mathrm{O}_{2}}$ was strengthened when the walk distance (6MWD) was multiplied by body weight (ie, walk work). ${ }^{16-18}$ Because the body weight factor may also influence STS and walk performances, a secondary aim of this study was to determine whether the aforementioned associations were strengthened when STS repetitions and 6MWD were expressed as a product of body weight.

\section{Methods}

\section{Study Population}

Twenty-five patients with CF attending the Renée Sabran Hospital Adult Cystic Fibrosis Service (regional CF center, CRCM Giens) were invited to participate in the study. Diagnosis of CF was based on clinical features and genotyping, according to standard guidelines. ${ }^{19}$ Subjects were at or close to their baseline clinical state at the time of the testing, as judged by the attending physician. They were not included if they (1) were $<18$ y old, (2) had unstable major non-pulmonary comorbidities, (3) were colonized with Burkholderia cepacia, (4) had contraindications for exercise testing, (5) had symptoms or signs of an acute pulmonary exacerbation, or (6) had received a lung transplant. Most of the subjects were physically active, being involved in non-hospital physical activities (eg, sports) and/or in-patient exercise rehabilitation programs. All refrained from strenuous physical exercise sessions on 


\section{1-Minute Sit-to-Stand in Adults With CF}

the days of testing. All were instructed to continue maintenance medications throughout the duration of their study involvement. Written informed consent was obtained, and the study was conducted according to the Declaration of Helsinki and approved by the institutional review board of the French Learned Society for Respiratory Medicine (institutional review board approval 2015-001).

\section{Experimental Design}

All subjects answered questionnaires and performed exercise tests in random order in $2 \mathrm{~d}$ over a period of 1 week. No tests were performed after CPET for a given day (ie, CPET was always the last test of the day). All tests were supervised by a trained operator.

\section{1-Minute Sit-to-Stand Test}

The STS test was performed using a chair $46 \mathrm{~cm}$ in height without arm rests, as described previously. ${ }^{20}$ Subjects were instructed to complete as many STS cycles as possible within $1 \mathrm{~min}$ at a self-paced speed. To fully stand up, the legs had to be straightened entirely (ie, complete knee extension), and to sit down, the chair had to be touched by the buttocks with the knees at approximately $90^{\circ}$ of flexion. To reduce the learning effect, the test was first demonstrated by the operator, and subjects had the opportunity to perform a few practice cycles to ensure correct realization. Standardized encouragement was given throughout the test.

\section{6-Minute Walk Test}

The 6MWT was performed indoors, along a flat straight 40-m corridor, according to the American Thoracic Society guidelines. ${ }^{21}$ Subjects were allowed to stop at any moment during the test but were told to walk as fast as they felt able to. At the end of the 6MWT, the total distance covered (6MWD) was recorded to the nearest meter.

\section{Quadriceps Force}

Quadriceps maximal isometric force was measured using a handheld dynamometer (MicroFet2, Hoggan Health Industries, Inc, West Jordan, Utah) with belt stabilization, as recommended. ${ }^{22}$ Subjects were tested in the sitting position with both knees and hips at $90^{\circ}$ of flexion. ${ }^{23}$ Subjects performed five 5-s maximal voluntary contractions of the dominant leg with a 1-min recovery period between attempts. Strong encouragement was given during each contraction. The first 2 attempts were excluded, and the best value among the remaining 3 attempts was retained for analysis. ${ }^{23}$ The moment arm was recorded, and strength was calculated in newton-meters.

\section{Pulmonary Function}

Pulmonary function data were obtained using spirometry measurements $\left(\mathrm{FEV}_{1}\right.$ and $\left.\mathrm{FVC}\right)$ and whole-body plethysmography (lung volumes) (MasterScreen Body plethysmograph, CareFusion, San Diego, California) (Table 1).

\section{Cardiopulmonary Exercise Test}

Subjects performed a continuous incremental cycle protocol to volitional exhaustion using an electronically braked cycle ergometer (Ergoselect 200, Ergoline, Bitz, Germany). They performed a 3-min warm-up at $20 \mathrm{~W}$, after which the work load was increased each minute. Increments (from 10 to $20 \mathrm{~W} / \mathrm{min}$ ) were chosen according to disease severity and sex, with the aim to obtain an exercise duration of $8-12 \mathrm{~min}$. Subjects were instructed to maintain a cadence of $60-70 \mathrm{rpm}$. They breathed through a mask that was connected to a calibrated metabolic cart (Masterscreen CPX, CareFusion, San Diego, California) permitting breath by breath gas exchange measurement (see supplementary materials at http://www.rcjournal.com).

\section{Questionnaires}

Habitual physical activity level was measured using the AQAP questionnaire. ${ }^{24} \mathrm{HRQOL}$ was assessed by the French version of the $\mathrm{CF}$ questionnaire for adults $(\mathrm{CFQ} 14+) .{ }^{25} \mathrm{~A}$ mean global score was calculated for both AQAP and CFQ14+. Self-esteem in the physical domain was assessed using the French version of the physical self-perception profile $^{26}$ (see supplementary material).

\section{Common Analysis for Exercise Tests}

Heart rate and $\mathrm{S}_{\mathrm{pO}_{2}}$ (Bluenight Oximeter, SleepInnov Technology, Moirans, France) were continuously monitored during the STS test, 6MWT, and CPET. The difference between resting $\mathrm{S}_{\mathrm{pO}_{2}}$ and minimum $\mathrm{S}_{\mathrm{pO}_{2}}$ was calculated as an index of exercise-induced oxygen desaturation. Exercise-induced hypoxemia was defined as a drop $>4 \%$, calculated as resting $\mathrm{S}_{\mathrm{pO}_{2}}-$ minimum $\mathrm{S}_{\mathrm{pO}_{2}}{ }^{1}$ Before and immediately at the end of each exercise test, subjects were asked to rate their sensations of breathlessness and muscle fatigue at peak exercise using the 15-point RPE (rated perceived exertion) scale. ${ }^{1,27}$ As previously suggested in patients with COPD, the 6MWD was multiplied by body weight to provide an index of walk work, which is an improved outcome measure for the 6MWT. ${ }^{16}$ STS repetitions were also expressed as a product of body weight, providing an index of total work and allowing optimal comparisons between the relationships given by $6 \mathrm{MWD} \times$ body weight and those given by STS repetitions $\times$ body weight. All main outcomes, when applicable, 


\section{1-Minute Sit-to-Stand in Adults With CF}

Table 1. Subjects' Baseline Characteristics and Questionnaires

\begin{tabular}{|c|c|c|}
\hline Variables & Values & Range \\
\hline Male/female sex, $n$ & $17 / 8$ & \\
\hline Age, y & $30 \pm 9$ & $18-45$ \\
\hline Height, m & $1.65 \pm 0.06$ & $1.51-1.76$ \\
\hline Body mass, $\mathrm{kg}$ & $55.6 \pm 6.2$ & $45.0-64.0$ \\
\hline BMI, $\mathrm{kg} / \mathrm{m}^{2}$ (\% underweight)* & $20.4 \pm 1.9(16)$ & $15.6-23.5$ \\
\hline Pancreatic insufficiency, $n(\%)$ & $22(88)$ & NA \\
\hline CF-related diabetes, $n(\%)$ & $7(28)$ & NA \\
\hline Antibiotic therapy/y, $n$ & $1.2 \pm 1.6$ & $0-7$ \\
\hline \multicolumn{3}{|l|}{ P. aeruginosa infection, $n$} \\
\hline Chronic & 23 & NA \\
\hline Intermittent & 0 & NA \\
\hline Never & 0 & NA \\
\hline Free & 2 & NA \\
\hline \multicolumn{3}{|l|}{ CFTR genotype, $n$} \\
\hline Homozygote $\Delta$ F508del & 12 & NA \\
\hline Heterozygote $\Delta$ F508del & 10 & NA \\
\hline $3659 \mathrm{delC}$ c. $3528 \mathrm{delC}$ p:1717-1G $>$ A c. $1585-1 \mathrm{G}>\mathrm{A}$ & 1 & NA \\
\hline $2183 \mathrm{AA}>\mathrm{G}: \mathrm{G} 542 \mathrm{X}$ & 1 & NA \\
\hline 1677delta/1677delta & 1 & NA \\
\hline \multicolumn{3}{|l|}{ Pulmonary function } \\
\hline $\mathrm{FEV}_{1}, \mathrm{~L}$ & $2.03 \pm 0.8$ & $0.76-3.4$ \\
\hline $\mathrm{FEV}_{1}, \%$ predicted & 59.5 & $21.8-112.0$ \\
\hline FVC, L & $3.25 \pm 0.9$ & $1.42-5.43$ \\
\hline FVC, $\%$ predicted & 80.5 & $42.3-125.1$ \\
\hline $\mathrm{FEV}_{1} / \mathrm{FVC}, \%$ & $60.8 \pm 11.0$ & $39.2-82.8$ \\
\hline $\mathrm{TGV}, \%$ predicted & $127 \pm 24$ & $92-175$ \\
\hline $\mathrm{RV}, \%$ predicted & $197 \pm 60$ & $105-336$ \\
\hline TLC, $\%$ predicted & $113 \pm 17$ & $85-146$ \\
\hline \multicolumn{3}{|l|}{ Questionnaires, median (IQR) } \\
\hline \multicolumn{3}{|l|}{ Physical activity level } \\
\hline Daily physical activity index & $2.8(2.0-3.1)$ & NA \\
\hline Sport index & $3.0(2.3-3.3)$ & NA \\
\hline Leisure-time index & $3.3(2.8-3.8)$ & NA \\
\hline Global score & $9.0(7.3-9.8)$ & NA \\
\hline \multicolumn{3}{|l|}{ HRQOL (CFQ14+) } \\
\hline Global score $(\%)$ & $68.8(56.3-73.8)$ & NA \\
\hline \multicolumn{3}{|l|}{ Physical self-perception profile } \\
\hline Global self-esteem & $4.6(3.4-5.2)$ & NA \\
\hline Physical self-worth & $3.6(2.8-4.4)$ & NA \\
\hline Endurance & $3.0(2.0-3.8)$ & NA \\
\hline Sports competence & $2.3(1.5-3.8)$ & NA \\
\hline Body appearance & $4.3(4.0-5.3)$ & NA \\
\hline Physical strength & $2.3(1.3-3.0)$ & NA \\
\hline $\begin{array}{l}\text { Data are given as mean } \pm \text { SD with the range also displayed (unless other } \\
\text { * Defined as BMI }<18.5 \mathrm{~kg} / \mathrm{m}^{2} . \\
\text { BMI }=\text { body mass index } \\
\text { NA = not applicable } \\
\text { CF }=\text { cystic fibrosis } \\
\text { CFTR = cystic fibrosis transmembrane conductance regulator } \\
\text { TGV = thoracic gas volume } \\
\mathrm{RV}=\text { residual volume } \\
\mathrm{TLC}=\text { total lung capacity } \\
\mathrm{IQR}=\text { interquartile range } \\
\mathrm{HRQOL}=\text { health-related quality of life } \\
\mathrm{CFQ}=\text { cystic fibrosis questionnaire }\end{array}$ & function data. Data are & questionnaires. \\
\hline
\end{tabular}




\section{1-Minute Sit-to-Stand in Adults With CF}

were expressed as percentages of predicted values (see supplementary material).

\section{Statistical Analysis}

Statistical analyses were conducted with Statistica 8 (StatSoft, Tulsa, Oklahoma). Normality of distribution and homogeneity of variances of main variables were verified using Shapiro-Wilk and Levene tests, respectively. Skewness was calculated as a measure of symmetry. Pearson correlations were calculated to investigate whether any relationship existed between main outcomes (ie, exercise and lung function parameters). Associations between questionnaire scores and main exercise parameters were tested by Spearman rank correlation analyses. When relevant, effect size for the difference between 2 significant correlations was calculated using Cohen's q with the following interpretation: $<0.10=$ no effect; $0.10-0.30=$ small effect; $0.30-0.50=$ medium effect; $>0.50=$ large effect. Repeated-measures analysis of variance and Tukey post hoc tests were performed to compare peak heart rate, oxygen desaturation, and peak scores of breathlessness and muscle fatigue between tests. All tests were 2-tailed, and statistical significance was set at $P<.05$. Results are given as mean $\pm \mathrm{SD}$ for baseline characteristics and exercise parameters. Questionnaire data, which should be considered as ordinal (ie, use of an arbitrary numerical scale for a given item), are given as median (interquartile range). The study was powered to detect a significant correlation of 0.65 or greater between STS repetitions and quadriceps force ${ }^{28}$ with an $\alpha$ risk of 0.01 and power of $1-$ $\beta=0.80$, requiring a minimum of 22 subjects. The study by Ozalevli et $\mathrm{al}^{28}$ was chosen because of its large sample size. Post hoc power analysis based on the primary outcome (ie, peak $\dot{\mathrm{V}}_{\mathrm{O}_{2}}$ ) was also computed using the findings by Radtke et al ${ }^{15}$ (see supplementary material).

\section{Results}

The main subject characteristics are shown in Table 1. All subjects $(N=25)$ completed the 4 tests and the 3 questionnaires. All performed CPET to a maximal or near maximal level according to the usual criteria (see supplementary material). Exercise data are shown in Table 2. STS repetitions were $71 \pm 12 \%$ predicted. The 6MWD and quadriceps force were $90 \pm 10$ and $93 \pm 29 \%$ predicted, respectively. Peak $\dot{\mathrm{V}}_{\mathrm{O}_{2}}$ and peak work rate during CPET were $62 \pm 16$ and $74 \pm 18 \%$ predicted, respectively. Peak heart rate during the STS test, CPET, and $6 \mathrm{MWT}$ were $71 \pm 9,93 \pm 7$, and $77 \pm 8 \%$ predicted, respectively.
Table 2. Subjects' Responses to Exercise Testing

\begin{tabular}{|c|c|c|}
\hline Exercise Test & Mean \pm SD & Range \\
\hline \multicolumn{3}{|l|}{ CPET } \\
\hline Peak work rate, $\mathrm{W}$ & $128 \pm 34$ & $70-185$ \\
\hline Peak work rate, $\mathrm{W} / \mathrm{kg}$ & $2.3 \pm 0.5$ & $1.32-3.08$ \\
\hline Peak $\dot{\mathrm{V}}_{\mathrm{O}_{2}}, \mathrm{~mL} / \mathrm{min}$ & $1,486 \pm 419$ & $730-2,277$ \\
\hline Peak $\dot{\mathrm{V}}_{\mathrm{O}_{2}}, \mathrm{~mL} / \mathrm{min}^{\prime} \mathrm{kg}$ & $26.6 \pm 6.5$ & $14.9-42.2$ \\
\hline Peak RER & $1.18 \pm 0.08$ & $1.02-1.34$ \\
\hline Peak $\dot{V}_{\mathrm{E}}, \mathrm{L} / \mathrm{min}$ & $63.2 \pm 19.2$ & $31-103$ \\
\hline$\dot{\mathrm{V}}_{\mathrm{E}} / \mathrm{MVV}, \%$ & $83.0 \pm 22.4$ & $45-133$ \\
\hline Peak heart rate, beats/min & $171 \pm 14$ & $143-192$ \\
\hline Oxygen desaturation, $\%$ & $7.4 \pm 5.1$ & $0-18$ \\
\hline Peak breathlessness & $17.6 \pm 2.1$ & $11-20$ \\
\hline Peak muscle fatigue & $17.9 \pm 1.9$ & $12-20$ \\
\hline \multicolumn{3}{|l|}{ STS test } \\
\hline Repetitions/min & $40 \pm 7$ & $29-56$ \\
\hline Repetitions $\times$ weight, $\mathrm{kg} / \mathrm{min}$ & $2,199 \pm 392$ & $1,519-2,898$ \\
\hline Peak heart rate, beats/min & $131 \pm 18$ & $100-182$ \\
\hline Oxygen desaturation, $\%$ & $3.7 \pm 2.9$ & $0-11$ \\
\hline Peak breathlessness & $12.8 \pm 2.6$ & $7-18$ \\
\hline Peak muscle fatigue & $12.0 \pm 3.5$ & $6-18$ \\
\hline \multicolumn{3}{|l|}{ 6MWT } \\
\hline 6MWD, m & $636 \pm 70$ & $514-780$ \\
\hline $6 \mathrm{MWD} \times$ weight, $\mathrm{kg} \mathrm{m}$ & $35,353 \pm 5,605$ & $24,075-43,800$ \\
\hline Peak heart rate, beats/min & $141 \pm 16$ & 119-178 \\
\hline Oxygen desaturation, $\%$ & $4.5 \pm 3.7$ & $0-17$ \\
\hline Peak breathlessness & $12.4 \pm 2.9$ & $7-20$ \\
\hline Peak muscle fatigue & $11.9 \pm 3.1$ & $6-17$ \\
\hline \multicolumn{3}{|l|}{ Quadriceps force } \\
\hline MVC, newton-m & $127 \pm 39$ & $50-227$ \\
\hline MVC, newtons & $407 \pm 116$ & $177-697$ \\
\hline $\begin{array}{l}\text { Data are given as mean } \pm \mathrm{SD} \text { with the range } \\
\mathrm{CPET}=\text { cardiopulmonary exercise test } \\
\dot{\mathrm{V}}_{\mathrm{O}_{2}}=\text { peak oxygen consumption } \\
\mathrm{RER}=\text { respiratory exchange ratio } \\
\dot{\mathrm{V}}_{\mathrm{E}}=\text { minute ventilation } \\
\mathrm{MVV}=\text { maximum voluntary ventilation } \\
\mathrm{STS}=\text { sit-to-stand } \\
6 \mathrm{MWT}=6 \text {-min walk test } \\
6 \mathrm{MWD}=6 \text {-min walk distance } \\
\mathrm{MVC}=\text { maximum voluntary contraction }\end{array}$ & so displayed unless oth & ise stated. \\
\hline
\end{tabular}

\section{Comparisons Between Tests}

Peak heart rate was lower during the STS test compared with CPET $(P<.001)$ and the 6MWT $(P=.009)$ (Table 2 ). No differences were found between sensations of breathlessness and muscle fatigue scores at peak exercise for each test ( $P$ ranging from .21 to .34). Both scores were similar at peak exercise between the STS test and the 6MWT (muscle fatigue, $P=.98$; breathlessness, $P=.77$ ) but were lower compared with scores obtained during CPET $(P<.001)$ (Table 2).

Oxygen desaturation during the STS test was lower compared with CPET $(P<.001)$ but not different compared with the 6MWT $(P=.59)$ (Table 2). Exercise-induced 
Table 3. Relationships Between STS Test and 6MWT and Main Study Outcomes

\begin{tabular}{|c|c|c|c|c|}
\hline Variables & $\begin{array}{c}\text { STS } \\
\text { Repetitions }\end{array}$ & 6MWD & $\begin{array}{c}\text { STS } \\
\text { Repetitions } \times \\
\text { Weight }\end{array}$ & $\begin{array}{c}\text { 6MWD } \times \\
\text { Weight }\end{array}$ \\
\hline \multicolumn{5}{|c|}{ Pulmonary function and physical fitness outcomes, Pearson correlation coefficients } \\
\hline $\mathrm{FEV}_{1}(\mathrm{~L})$ & 0.16 & $0.47 *$ & 0.28 & $0.46^{*}$ \\
\hline $\mathrm{FEV}_{1}(\%$ predicted $)$ & 0.20 & 0.39 & 0.18 & 0.24 \\
\hline Peak work rate $(\mathrm{W})$ & 0.15 & $0.54 \dagger$ & $0.48 *$ & $0.76 \neq$ \\
\hline Peak $\dot{\mathrm{V}}_{\mathrm{O}_{2}}\left(\mathrm{~mL} \mathrm{~min}^{-1}\right)$ & 0.20 & $0.46^{*}$ & $0.56 \dagger$ & $0.73 \ddagger$ \\
\hline Peak $\dot{\mathrm{V}}_{\mathrm{O}_{2}}(\mathrm{~mL} / \mathrm{min} / \mathrm{kg})$ & 0.38 & $0.53 \dagger$ & $0.52 \dagger$ & $0.54 \dagger$ \\
\hline Quadriceps MVC (N) & 0.17 & 0.17 & $0.52 \dagger$ & $0.51 \dagger$ \\
\hline \multicolumn{5}{|c|}{ Questionnaires, Spearman rank correlation coefficients } \\
\hline PA level (global score) & 0.24 & $0.48 *$ & $0.46^{*}$ & $0.53 \dagger$ \\
\hline HRQOL (global score) & 0.22 & 0.20 & 0.37 & $0.41^{*}$ \\
\hline Global self-esteem & 0.08 & 0.22 & 0.39 & $0.49^{*}$ \\
\hline Physical self-worth & 0.26 & 0.27 & $0.52 \dagger$ & $0.48^{*}$ \\
\hline Endurance & 0.23 & $0.49^{*}$ & 0.39 & $0.53 \dagger$ \\
\hline Sports competence & 0.16 & $0.48 *$ & $0.58 \dagger$ & $0.74+$ \\
\hline Body appearance & 0.06 & -0.11 & 0.24 & 0.17 \\
\hline Physical strength & $0.41^{*}$ & 0.18 & $0.67 \ddagger$ & $0.47^{*}$ \\
\hline $\begin{array}{l}\text { Data are Pearson correlation coefficients for pulmonary functic } \\
* P<.05 \\
\dagger P<.01 \\
\ddagger P<.001 \\
\text { STS }=\text { sit-to-stand } \\
\text { 6MWD = 6-min walk distance } \\
\mathrm{V}_{\mathrm{O}_{2}}=\text { peak oxygen consumption } \\
\text { MVC = maximum voluntary contraction } \\
\text { PA = physical activity } \\
\text { HRQOL = health-related quality of life }\end{array}$ & coefficients for & ionnaires. & & \\
\hline
\end{tabular}

hypoxemia was detected in 9 subjects for the STS test, 11 subjects for the 6MWT, and 15 subjects for CPET. $89 \%$ of subjects with a drop $>4 \%$ of the resting $\mathrm{S}_{\mathrm{pO}_{2}}$ during STS test had minimal $\mathrm{S}_{\mathrm{pO}_{2}}$ of $<90 \%$ during CPET. $91 \%$ of subjects with a drop $<4 \%$ of the resting $\mathrm{S}_{\mathrm{pO}_{2}}$ during the STS test had minimal $\mathrm{S}_{\mathrm{pO}_{2}}$ of $>90 \%$ during CPET.

\section{Relationship Between Tests}

Correlation analyses are shown in Table 3. STS repetitions tended to be associated with peak $\dot{\mathrm{V}}_{\mathrm{O}_{2}}$ (in $\mathrm{mL} \mathrm{min}{ }^{-1}$ $\mathrm{kg}^{-1}, P=.062$ ), without significant associations with other main outcomes except for perceived physical strength $(P=.044)$ (Table 3$)$. When expressed as a product of body weight, STS repetitions had significant associations with quadriceps strength, peak $\dot{\mathrm{V}}_{\mathrm{O}_{2}}$, peak work rate, and self-esteem (Table 3 ). STS repetitions $\times$ body weight had lower associations with peak $\dot{\mathrm{V}}_{\mathrm{O}_{2}}$ (in $\mathrm{mL} \mathrm{min}{ }^{-1}$ ) and peak work rate than 6MWD $\times$ body weight (Cohen's $q=0.30$ [small effect] and 0.47 [medium effect], respectively).

Oxygen desaturation during the STS test was strongly associated with oxygen desaturation during CPET $(r=0.80$, $P<.001$, Fig. 1). This association was better than the

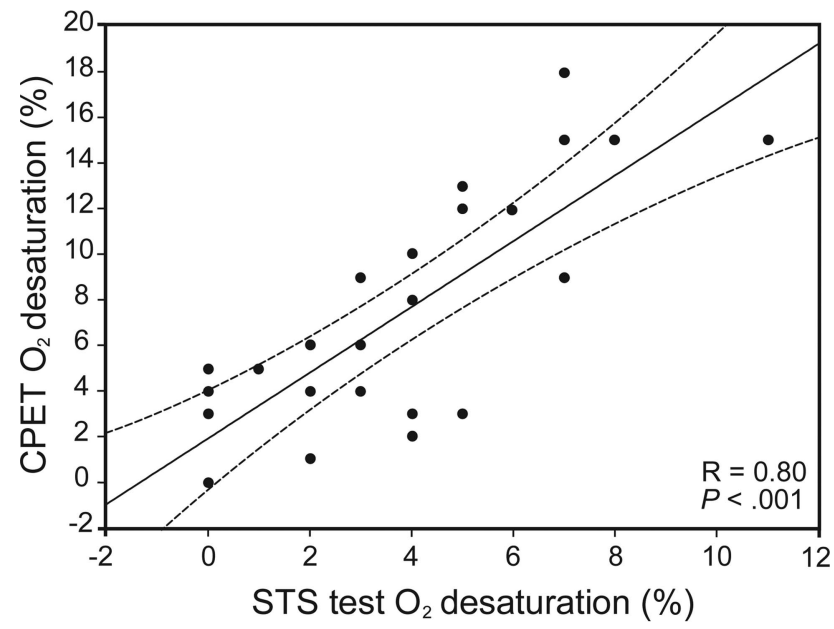

Fig. 1. Relationship between oxygen desaturation during sit-tostand (STS) test and oxygen desaturation during cardiopulmonary exercise test (CPET). For both tests, oxygen desaturation was calculated as the difference between resting $\mathrm{S}_{\mathrm{pO}_{2}}$ and minimum $\mathrm{S}_{\mathrm{pO}_{2}}$ reached during the test. The solid line is the best least-square fit correlation. Curved dashed lines show $95 \% \mathrm{Cl}$.

association between oxygen desaturation during the 6MWT and oxygen desaturation during CPET (Cohen's q $=0.64$, large effect). 


\section{1-Minute Sit-to-Stand in Adults With CF}

$6 \mathrm{MWD} \times$ body weight had stronger associations with peak $\dot{\mathrm{V}}_{\mathrm{O}_{2}}$ (in $\mathrm{mL} \mathrm{min}^{-1}$ ) and peak work rate than 6MWD alone (Cohen's q $=0.43$ and 0.39 , respectively, both medium effect). Peak $\dot{\mathrm{V}}_{\mathrm{O}_{2}}$ and peak work rate during CPET had the greatest number of significant associations with items of HRQOL and physical self-perception ( $\mathrm{r}$ range 0.41-0.80; see supplementary material).

\section{Discussion}

We found that (1) the STS is a submaximal test, as indicated by submaximal perceived symptoms and cardiac demand; (2) the STS test had positive associations with indices of peak aerobic capacity, quadriceps strength, and self-esteem but only when STS repetitions were expressed as a product of body weight; (3) these associations were weaker than those obtained from $6 \mathrm{MWD} \times$ weight; and (4) oxygen desaturation during the STS test was strongly associated with oxygen desaturation during CPET.

STS repetitions were $71 \%$ of predicted in our CF population, suggesting the sensitivity of this test to detect reduced physical fitness. Even though we did not include a control group of healthy subjects, it is intuitive that the large sample from which the equation used in our study was derived ${ }^{20}$ should provide a more accurate reference than a smaller number of matched controls. Reduced STS performance was also consistently found in various chronic diseases, including COPD. ${ }^{28}$ STS performance in 14 adults with $\mathrm{CF}$ in the study by Radtke et $\mathrm{al}^{15}$ was $>75$ th percentile according to reference values. ${ }^{20}$ Even if within-subject comparison may not be much affected in cross-sectional (eg, relationship with other tests) or longitudinal designs (ie, STS performance pre-post intervention) for a given protocol, comparison between studies may be limited by differences in subject characteristics and protocol modalities (eg, nature and frequency of verbal encouragement). Consensus guidelines for the full standardization of the STS test are therefore needed to allow optimal betweenstudies comparisons.

To be clinically meaningful, the STS test should demonstrate significant associations with main outcomes of the accepted standard exercise test (ie, CPET). Moreover, if STS is intended to be used when CPET is not available or cannot be performed (eg, in some severe patients), then it should have comparable associations with CPET outcomes as do conventional field tests, such as the 6MWT. Previous studies in COPD ${ }^{16-18}$ suggested that the work calculated as the product of $6 \mathrm{MWD} \times$ body weight was an improved outcome measure compared with 6MWD. Our results, showing 6MWD $\times$ body weight as the strongest correlate of peak $\dot{\mathrm{V}}_{\mathrm{O}_{2}}$, clearly extend this finding to the adult $\mathrm{CF}$ population. Although the associations were weaker, we found positive correlations between STS repetitions $\times$ body weight and indices of peak aerobic capac- ity. In a practical perspective, our results suggest that if both the 6MWT and CPET cannot be included in a routine basis, then STS repetitions $\times$ body weight outcome might be a submaximal alternative to reflect the physical fitness of a patient with CF. This alternative may be particularly attractive for regular assessment of severe patients because the STS test produces less hemodynamic stress compared with 6MWT and CPET.

Limb muscle testing is of clinical importance in $\mathrm{CF}$ because muscle weakness is prevalent in this population ${ }^{9}$ and is associated with exercise intolerance ${ }^{10}$ and reduced HRQOL. ${ }^{5}$ Based on significant associations with quadriceps strength, ${ }^{12,28}$ the STS test has been proposed as a simple measure of lower-limb strength. We expressed STS repetitions as a product of body weight because this factor clearly influences STS performance. We found only a moderate correlation between STS repetitions and quadriceps strength. Compared with previous studies in COPD,,$^{12,28}$ our subjects had relatively preserved lower-limb strength, and much lower inter-individual variability was found for STS performance and quadriceps strength, thereby potentially weakening the association between these parameters. Moreover, previous studies did not use a reference method to evaluate limb muscle strength (eg, manual force testing $^{28}$ or one-repetition maximum ${ }^{12}$ ), potentially leading to inaccuracies affecting the relationship with STS performance. Overall, the moderate association between the STS test and quadriceps force in our study supports their convergent validity. However, the magnitude of the correlation is clearly inadequate to claim that these 2 measures are merely describing the same construct in CF. This also suggests that other factors probably contribute to STS performance in $\mathrm{CF}$, such as postural balance and quadriceps endurance, both of which may be impaired in some patients. ${ }^{10,29}$ Further studies are necessary to identify the factors explaining reduced STS performance in patients with CF. It would also be of value to determine whether STS repetitions are positively associated with anaerobic capacity, an important contributor of many daily life activities.

Possibly because maximal exercise involves the integrated functioning of multiple organs in multisystem diseases, such as CF, peak $\dot{\mathrm{V}}_{\mathrm{O}_{2}}$ has been demonstrated as a strong predictor of HRQOL. 5 Our findings are in line with this study and extend this association to measures of selfesteem, which is also compromised in CF. ${ }^{30}$ These findings underline the importance of a high level of peak aerobic capacity as a contributor to psychological and sociological well-being. We found that both STS repetitions and 6MWD, even when expressed as a product of body weight, had only minor positive associations with HRQOL and self-esteem. Thus, it can be speculated that longitudinal improvement in submaximal indices of fitness, without concomitant improvement in peak aerobic 


\section{1-Minute Sit-to-Stand in Adults With CF}

capacity, should not transfer to psychological and/or sociological benefits in patients with CF.

Exercise-induced oxygen desaturation is common in patients with $\mathrm{CF}$ and cannot be accurately predicted from resting $\mathrm{S}_{\mathrm{pO}_{2}}$ and $\mathrm{FEV}_{1} \cdot{ }^{3}$ CPET is thus recommended to identify exercise-induced hypoxemia. ${ }^{1}$ Detecting exerciseinduced oxygen desaturation and particularly patients with a drop of $\mathrm{S}_{\mathrm{pO}_{2}}$ to $<90 \%$ is of clinical importance because it should guide the treatment for hypoxemia (ie, supplemental oxygen). ${ }^{1}$ Here, we found a strong correlation between oxygen desaturation during STS test and oxygen desaturation during CPET and found that $\sim 90 \%$ of subjects with significant oxygen desaturation during the STS test had a drop of $\mathrm{S}_{\mathrm{pO}_{2}}$ to $<90 \%$ during CPET. Thus, patients exhibiting significant oxygen desaturation during the STS test may be at risk for severe hypoxemia, reinforcing the rationale for performing CPET. This finding will require confirmation in a larger group of subjects.

There were some limitations to this study, potentially inducing variability in the data and limiting comparison with other studies. First, due to logistical and methodological considerations, it was impossible to conduct a true randomized counterbalanced design. Second, except for quadriceps force assessment, each test was performed on just one occasion for each subject (ie, cross-sectional), and performance in some tests may have been be slightly affected by daily changes in mucus accumulation and adherence to medications. Last, even if all of these tests have previously demonstrated satisfactory reliability, a learning effect may be present for some tests, and performances may have been slightly underestimated.

\section{Conclusions}

The STS test cannot be used as a substitute for CPET to accurately assess maximal exercise tolerance in adults with CF. The STS test may be useful in detecting patients with CF who may exhibit a high level of oxygen desaturation during heavy exercise. Considering its low hemodynamic demand and its significant associations with important physiological and psychological outcomes, STS repetitions $\times$ body weight may still be an interesting submaximal parameter. However, further studies are mandatory to specify the role of additional factors, such as postural balance and quadriceps endurance, in explaining STS performance in $\mathrm{CF}$.

\section{REFERENCES}

1. Hebestreit H, Arets HG, Aurora P, Boas S, Cerny F, Hulzebos EH, et al. Statement on exercise testing in cystic fibrosis. Respiration 2015;90(4):332-351.

2. Moran F, Bradley J. Incorporating exercise into the routine care of individuals with cystic fibrosis: is the time right? Expert Rev Respir Med 2010;4(2):139-142.
3. Ruf K, Hebestreit H. Exercise-induced hypoxemia and cardiac arrhythmia in cystic fibrosis. J Cyst Fibros 2009;8(2):83-90.

4. Nixon PA, Orenstein DM, Kelsey SF, Doershuk CF. The prognostic value of exercise testing in patients with cystic fibrosis. N Engl J Med 1992;327(25):1785-1788.

5. Hebestreit H, Schmid K, Kieser S, Junge S, Ballmann M, Roth K, et al. Quality of life is associated with physical activity and fitness in cystic fibrosis. BMC Pulm Med 2014;14:26.

6. Stevens D, Oades PJ, Armstrong N, Williams CA. A survey of exercise testing and training in UK cystic fibrosis clinics. J Cyst Fibros 2010;9(5):302-306.

7. Gruet M, Brisswalter J, Mely L, Vallier JM. Use of the peak heart rate reached during six-minute walk test to predict individualized training intensity in patients with cystic fibrosis: validity and reliability. Arch Phys Med Rehabil 2010;91(4):602-607.

8. Martin C, Chapron J, Hubert D, Kanaan R, Honoré I, Paillasseur JL, et al. Prognostic value of six minute walk test in cystic fibrosis adults. Respir Med 2013;107(12):1881-1887.

9. Troosters T, Langer D, Vrijsen B, Segers J, Wouters K, Janssens W, et al. Skeletal muscle weakness, exercise tolerance and physical activity in adults with cystic fibrosis. Eur Respir J 2009;33(1):99-106.

10. Gruet M, Decorte N, Mely L, Vallier JM, Camara B, Quetant S, et al. Skeletal muscle contractility and fatigability in adults with cystic fibrosis. J Cyst Fibros 2016;15(1):e1-e8.

11. Klijn PH, Oudshoorn A, van der Ent CK, van der Net J, Kimpen JL, Helders PJ. Effects of anaerobic training in children with cystic fibrosis: a randomized controlled study. Chest 2004;125(4):12991305.

12. Zanini A, Aiello M, Cherubino F, Zampogna E, Azzola A, Chetta A, Spanevello A. The one repetition maximum test and the sit-to-stand test in the assessment of a specific pulmonary rehabilitation program on peripheral muscle strength in COPD patients. Int $\mathrm{J}$ Chron Obstruct Pulmon Dis 2015;10:2423-2430.

13. Puhan MA, Siebeling L, Zoller M, Muggensturm P, ter Riet G. Simple functional performance tests and mortality in COPD. Eur Respir J 2013;42(4):956-963.

14. Meriem M, Cherif J, Toujani S, Ouahchi Y, Hmida AB, Beji M. Sit-to-stand test and 6-min walking test correlation in patients with chronic obstructive pulmonary disease. Ann Thorac Med 2015;10(4): 269-273.

15. Radtke T, Puhan MA, Hebestreit H, Kriemler S. The 1-min sit-tostand test: a simple functional capacity test in cystic fibrosis? J Cyst Fibros 2016;15(2):223-226.

16. Carter R, Holiday DB, Nwasuruba C, Stocks J, Grothues C, Tiep B. 6-minute walk work for assessment of functional capacity in patients with COPD. Chest 2003;123(5):1408-1415.

17. Chuang ML, Lin IF, Wasserman K. The body weight-walking distance product as related to lung function, anaerobic threshold and peak $\mathrm{VO}_{2}$ in COPD patients. Respir Med 2001;95(7):618-626.

18. Poersch K, Berton DC, Canterle DB, Castilho J, Lopes AL, Martins $\mathrm{J}$, et al. Six-minute walk distance and work relationship with incremental treadmill cardiopulmonary exercise test in COPD. Clin Respir J 2013;7(2):145-152.

19. Farrell PM, Rosenstein BJ, White TB, Accurso FJ, Castellani C, Cutting GR, et al. Guidelines for diagnosis of cystic fibrosis in newborns through older adults: Cystic Fibrosis Foundation consensus report. J Pediatr 2008;153(2):S4-S14.

20. Strassmann A, Steurer-Stey C, Lana KD, Zoller M, Turk AJ, Suter $\mathrm{P}$, Puhan MA. Population-based reference values for the 1-min sitto-stand test. Int J Public Health 2013;58(6):949-953.

21. ATS Committee on Proficiency Standards for Clinical Pulmonary Function Laboratories: ATS statement: guidelines for the six-minute walk test. Am J Respir Crit Care Med 2002;166(1):111-117. 


\section{1-Minute Sit-to-Stand in Adults With CF}

22. Bachasson D, Villiot-Danger E, Verges S, Hayot M, Perez T, Chambellan A, Wuyam B. [Maximal isometric voluntary quadriceps strength assessment in COPD]. Rev Mal Respir 2014;31(8):765-770.

23. Maltais F, Decramer M, Casaburi R, Barreiro E, Burelle Y, Debigaré $\mathrm{R}$, et al. An official American Thoracic Society/European Respiratory Society statement: update on limb muscle dysfunction in chronic obstructive pulmonary disease. Am J Respir Crit Care Med 2014; 189(9):e15-e62.

24. Vol S, Bedouet M, Gusto G, Leglu C, Beslin E, Decou P, et al. Evaluating physical activity: the AQAP questionnaire and its interpretation software. Ann Phys Rehabil Med 2011;54(8):478-495.

25. Henry B, Aussage P, Grosskopf C, Goehrs JM. Development of the Cystic Fibrosis Questionnaire (CFQ) for assessing quality of life in pediatric and adult patients. Qual Life Res 2003;12(1):63-76.
26. Ninot G, Delignières D, Fortes M. L'évaluation de l'estime de soi dans le domaine corporel. STAPS 2000;53:35-48.

27. Borg G. Borg's perceived exertion and pain scales. Champaign, Illinois: Human Kinetics; 1998.

28. Ozalevli S, Ozden A, Itil O, Akkoclu A. Comparison of the sit-tostand test with 6 min walk test in patients with chronic obstructive pulmonary disease. Respir Med 2007;101(2):286-293.

29. Lima TR, Guimãraes FS, Sá Ferreira A, Penafortes JT, Almeida VP, Lopes AJ. Correlation between posture, balance control, and peripheral muscle function in adults with cystic fibrosis. Physiother Theory Pract 2014;30(2):79-84.

30. Platten MJ, Newman E, Quayle E. Self-esteem and its relationship to mental health and quality of life in adults with cystic fibrosis. J Clin Psychol Med Settings 2013;20(3):392-399. 